\title{
Electrophysiologic Study and Ablation in Nepal - Our Initial Experience
}

\section{Rajbhandari Sujeeb, K.C, Man Bahadur, Sharma D, Shrestha MP Naik Ajaya}

Cardiac electrophysiology study (EPS) is one of the most advanced, challenging and a complex branch of invasive cardiology. It involves placement of large sized multipolar catheters in various chambers of the heart. They are used to map and also to ablate the culprit focus of arrhythmia generation. Supraventricular arrhythmias are a nuisance as the pharmacological treatment is only suppressive. When done by experts, the procedure can be curative. During the month of October 03, twelve cases were selected for EPS. There listed below.

\begin{tabular}{|l|l|l|l|l|}
\hline No. & Age/Sex & Diagnosis & Findings & Procedure \\
\hline 1 & $20 / F e$ & WPW & Right Free wall Accessory Pathway & Ablation \\
\hline 2 & $55 / F e$ & SVT & Typical AVNRT & Ablation \\
\hline 3 & $78 / F e$ & SVT & Typical AVNRT & Ablation \\
\hline 4 & $52 / \mathrm{M}$ & WPW & Right Posterior Accessory Pathway & Ablation \\
\hline 5 & $58 / \mathrm{Fe}$ & WPW & Right Free Wall Pathway & Ablation \\
\hline 6 & $39 / \mathrm{M}$ & SVT & Concealed left Lateral Pathway & Ablation \\
\hline 7 & $30 / \mathrm{Fe}$ & SVT & Typical AVNRT & Ablation \\
\hline 8 & $29 / \mathrm{Fe}$ & SVT & Concealed Left Lateral Pathway & Ablation \\
\hline 9 & $38 / \mathrm{Fe}$ & WPW & Left Lateral Accessory Pathway & Ablation \\
\hline 10 & $17 / \mathrm{Fe}$ & WPW & $\begin{array}{l}\text { i) Right Posterior Accessory Pathway } \\
\text { ii) Right Lateral Free Wall Accessory }\end{array}$ & Ablation \\
\hline 11 & $86 / \mathrm{M}$ & & $\begin{array}{l}\text { Fathway } \\
\text { HV interval normal (38ms) Prolonged }\end{array}$ & \\
\hline 12 & $68 / \mathrm{M}$ & SSS & AH (170 ms) Prolonged PR & SNRT- normal \\
\hline
\end{tabular}

Out of the twelve selected cases ten had radio frequency ablation and two had electrophoresis study done. Out of the ten only one had reappearance of delta wave on the ECG, In conclusion, this is an emerging field in Nepal and should be encouraged, 